\title{
Correction to: Deep In-memory Architectures for Machine Learning
}

\section{Correction to:}

M. Kang et al., Deep In-memory Architectures

for Machine Learning,

https://doi.org/10.1007/978-3-030-35971-3

This book was inadvertently published with an incorrect affiliation of the author "Mingu Kang". The affiliation details have been corrected now. 